\title{
Microplastic in the gastrointestinal tract of fishes along the Saudi Arabian Red Sea coast
}

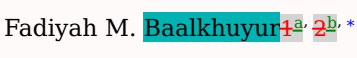

fadiyah.baalkhuyur@kaust.edu.sa

El-Jawaher A. Bin Dohaish1

Manal E.A. Elhalwagy $3^{\circ}$

Nabeel M. Alikunhizb

Abdulaziz M. AlSuwailem $4^{\complement}$

Anders Røstad $z^{\underline{b}}$

Darren J. Cokerz

Michael L. Berumenz-

Carlos M. Duarte $2^{\underline{B}}$

프King Abdulaziz University (KAU), Al Faisaliah Branch, Department of Zoology, Jeddah 51459-21453, Saudi Arabia

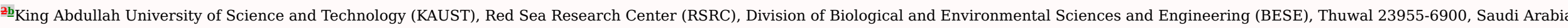

${ }^{36}$ King Abdulaziz University (KAU), Al Faisaliah Branch, Department of Biochemistry, Jeddah 51459-21453, Saudi Arabia

${ }^{4 d}$ King Abdullah University of Science and Technology (KAUST), Beacon Development Company, Thuwal 23955-6900, Saudi Arabia

${ }^{*}$ Corresponding author at: King Abdulaziz University (KAU), Al Faisaliah Branch, Department of Zoology, Jeddah 51459-21453, Saudi Arabia.

Abstract

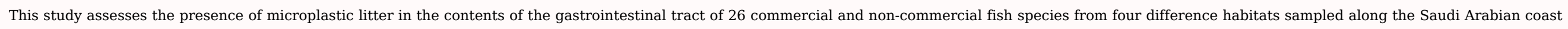

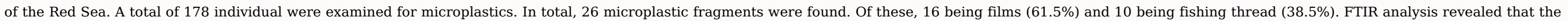

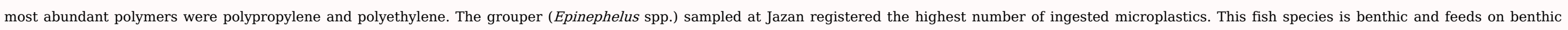

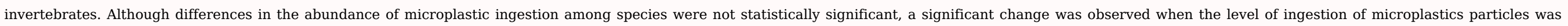

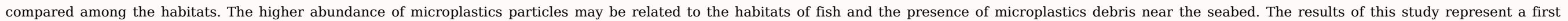
evidence that microplastic pollution represents an emerging threat to Red Sea fishes, their food web and human consumers.

Keywords: Stomach content; FT-IR; Polymer; Commercial fish; Grouper; Mesopelagic fish

\subsection{Introduction}

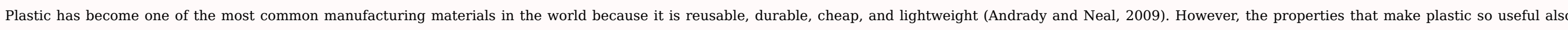

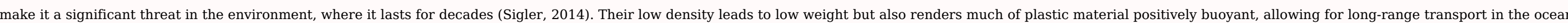

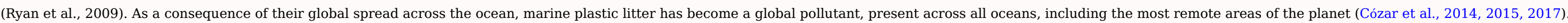




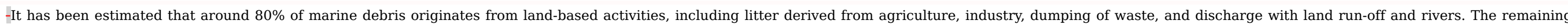

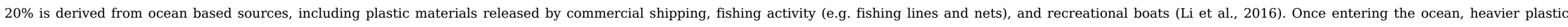

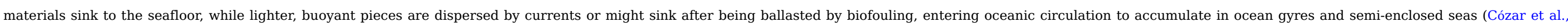
2014, 2015, 2017).

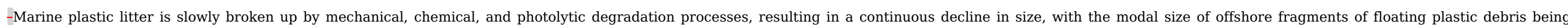

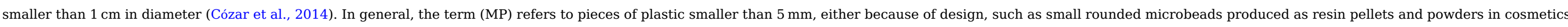

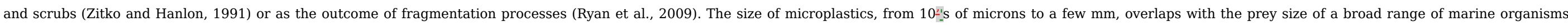

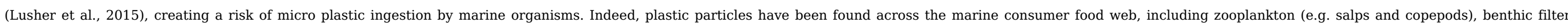

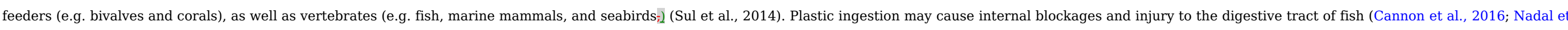

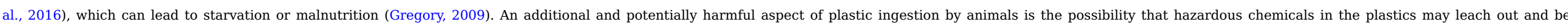
absorbed into the animalis body. This could potentially cause toxic effects to the animal (Rochman et al., 2015).

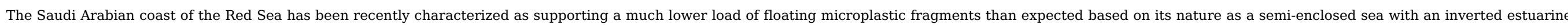

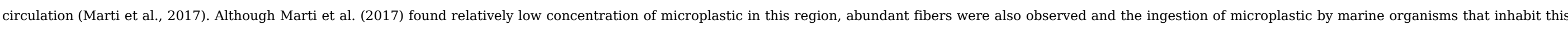

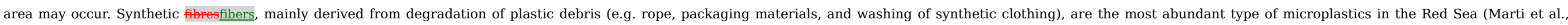

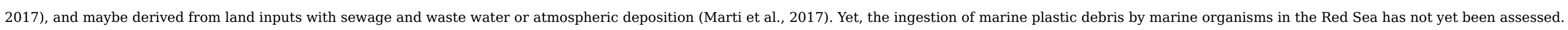

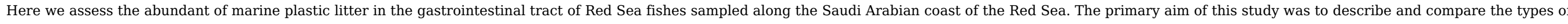

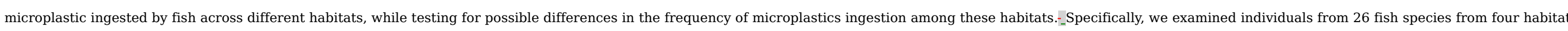
types: demersal, seagrass, coral reef, and mesopelagic habitats.

\subsection{Materials and methods}

\subsubsection{Sampling}

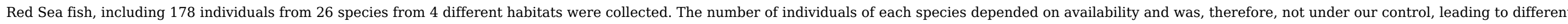

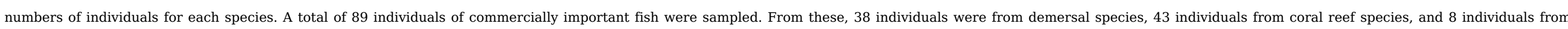

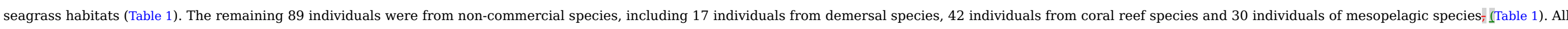
individuals, from commercial and non-commercial species, were sampled from seven locations along the Saudi Arabian Red Sea coast (Fig. 1, Table 1).

Table 1 Mean values and range of fish length, weight, and stomach weight for all species, (n)_= number of fish collected. alt-text: Table 1

\begin{tabular}{|c|c|c|c|c|c|c|c|c|c|c|}
\hline Species name & Species common__name & Habitat & Location & $\begin{array}{l}\text { Commercial } \\
\text { Yes/No }\end{array}$ & Sample-_(n) & $\begin{array}{l}\text { Mean length } \\
(\mathrm{cm}) \pm \mathrm{SD}\end{array}$ & $\begin{array}{c}\text { Length_range } \\
(\mathrm{cm})\end{array}$ & $\begin{array}{l}\text { Mean weight } \\
\quad(\mathrm{g}) \pm \mathrm{SD}\end{array}$ & $\begin{array}{l}\text { Weight range } \\
(\mathrm{g})\end{array}$ & $\begin{array}{c}\text { Mean stomach weight } \\
\text { (g) } \pm \text { SD }\end{array}$ \\
\hline Acanthurus gahhm & Black surgeonfish & Demersal & Jizan & Yes & 10 & $33.82 \pm 3.72$ & $40--28.1$ & $535.1 \pm 187.97$ & 848---_308 & $21.27 \pm 6.71$ \\
\hline Pristipomoides typus & White snapper & Demersal & Jizan & NO & 5 & $28.46 \pm 2.46$ & $32.1--25.7$ & $252.2 \pm 70.08$ & 368----188 & $3.71 \pm 0.48$ \\
\hline Epinephelus areolatus & Areolate grouper & Seagrass & Jizan & Yes & 5 & $28.42 \pm 4.24$ & $33.7-\cdots=23.8$ & $281.6 \pm 127.85$ & $447---175$ & $9.43 \pm 1.29$ \\
\hline $\begin{array}{l}\text { Pristipomoides } \\
\text { multidens }\end{array}$ & Goldbanded jobfish & Demersal & Jizan/Qahmah & Yes & 10 & $28.2 \pm 2.66$ & $33.2--=25.5$ & $236.7 \pm 66.26$ & 364----185 & $5.47 \pm 2.19$ \\
\hline Lutjanus kasmira & Bluestripe snapper & Coral reef & Jizan & Yes & 12 & $24.45 \pm 3.77$ & $34.9--=20.3$ & $233.17 \pm 143.5$ & $665--=121$ & $4.22 \pm 3.52$ \\
\hline
\end{tabular}




\begin{tabular}{|c|c|c|c|c|c|c|c|c|c|c|}
\hline Lethrinus microdon & Smalltooth emperor & Coral reef & Jizan & Yes & 10 & $29.53 \pm 5.2$ & $38.5--=22.7$ & $316.5 \pm 146.22$ & $605---147$ & $6.66 \pm 2.52$ \\
\hline $\begin{array}{l}\text { Epinephelus } \\
\text { chlorostigma }\end{array}$ & Brownspotted grouper & Seagrass & Jizan & Yes & 3 & $36.33 \pm 9.92$ & $42.7--=24.9$ & $700.33 \pm 443.36$ & 1019 & $12.27 \pm 5.87$ \\
\hline $\begin{array}{l}\text { Gymnocranius } \\
\text { grandoculis }\end{array}$ & $\begin{array}{l}\text { Bluelined large-eye } \\
\text { bream }\end{array}$ & Coral reef & Jizan & No & 10 & $28.23 \pm 2.31$ & $33.1--=26.1$ & $344 \pm 110.16$ & $609---244$ & $5.21 \pm 2.32$ \\
\hline Parascolopsis eriomma & $\begin{array}{l}\text { Rosy dwarf monocle } \\
\text { bream }\end{array}$ & Demersal & Jizan & Yes & 5 & $23.3 \pm 1.13$ & $24.8---22.2$ & $171 \pm 21.37$ & $200--=147$ & $4.13 \pm 2.41$ \\
\hline $\begin{array}{l}\text { Sargocentron } \\
\text { spiniferum }\end{array}$ & Sabre squirrelfish & Coral reef & Qahmah & NO & 5 & $30.68 \pm 0.89$ & $32--30.1$ & $427 \pm 45.39$ & 505----394 & $11.8 \pm 3.11$ \\
\hline Epinephelus radiatus & $\begin{array}{l}\text { Oblique-banded } \\
\text { grouper }\end{array}$ & Demersal & Qahmah & NO & 7 & $29.34 \pm 3.33$ & $34.6--=25.2$ & $359.29 \pm 129.06$ & $582---=217$ & $9.14 \pm 3.13$ \\
\hline $\begin{array}{l}\text { Lipocheilus } \\
\text { carnolabrum }\end{array}$ & Tang's snapper & Demersal & Qahmah & Yes & 7 & $24.39 \pm 3.9$ & $31.6--=20.7$ & $214 \pm 120.32$ & $444--=117$ & $4.29 \pm 2.56$ \\
\hline $\begin{array}{l}\text { Plectorhinchus } \\
\text { gaterinus }\end{array}$ & Blackspotted rubberlip & Demersal & Qahmah & Yes & 6 & $26.53 \pm 1.96$ & $29.5---24.2$ & $235.17 \pm 46.23$ & 298---181 & $6.33 \pm 3.44$ \\
\hline Epinephelus epistictus & Dotted grouper & Demersal & Jizan & NO & 5 & $31.4 \pm 6.9$ & $38--21.5$ & $424.4 \pm 231.23$ & $716---=148$ & $9.2 \pm 3.7$ \\
\hline Pygoplites diacanthus & Royal angelfish & Coral reef & $\begin{array}{l}\text { Offshore } \\
\text { KAUST }\end{array}$ & No & 5 & $14.06 \pm 2.55$ & $17--=10$ & $74 \pm 21.82$ & $99--47$ & $7.6 \pm 2.07$ \\
\hline Cephalopholis argus & Peacock hind & Coral reef & Yanbu & Yes & 4 & $23.63 \pm 1.3$ & $25.5--=22.5$ & $201 \pm 43.64$ & 266----172 & $7.25 \pm 5.85$ \\
\hline $\begin{array}{l}\text { Abudefduf } \\
\text { sexfasciatus }\end{array}$ & Scissortail sergeant & Coral reef & Al-Lith & No & 5 & $14.63 \pm 0.63$ & $15.5-=14$ & $60.8 \pm 5.4$ & $67-=55$ & $1.35 \pm 0.43$ \\
\hline Acanthurus sohal & Red Sea surgeonfish & Coral reef & Al-Lith & Yes & 3 & $18.9 \pm 3.29$ & $21.5-15.2$ & $92 \pm 37.04$ & $128---54$ & $3.67 \pm 1.15$ \\
\hline Dascyllus trimaculatus & Threespot dascyllus & Coral reef & Al-Lith & No & 2 & $10.5 \pm 0.71$ & $11-10$ & $32.5 \pm 0.71$ & $33-=32$ & $1 \pm 0$ \\
\hline Chaetodon austriacus & Blacktail butterflyfish & Coral reef & Duba & No & 10 & $10.82 \pm 0.44$ & $11.5--=10$ & $34.8 \pm 3.94$ & $39--\_26$ & $1.1 \pm 0.57$ \\
\hline Neoniphon sammara & Sammara squirrelfish & Coral reef & Al-Lith & No & 5 & $15.62 \pm 1.64$ & $18.2--=13.8$ & $31.2 \pm 6.65$ & $37---23$ & $1.6 \pm 0.55$ \\
\hline Naso unicornis & Bluespine unicornfish & Coral reef & $\begin{array}{l}\text { Offshore } \\
\text { KAUST }\end{array}$ & Yes & 2 & $40 \pm 2.83$ & $42--38$ & $901 \pm 70.71$ & $951---851$ & $130 \pm 7.07$ \\
\hline Thalassoma rueppellii & Klunzinger's wrasse & Coral reef & Al-Lith & Yes & 12 & $16.12 \pm 1.75$ & $19.5-=14$ & $49.25 \pm 17.7$ & $85--23$ & $1.33 \pm 0.65$ \\
\hline Benthosema pterotum & $\begin{array}{l}\text { Skinnycheek } \\
\text { lanternfish }\end{array}$ & Mesopelagic & KAEC & No & 10 & $0 \pm 0$ & $2.5=1.9$ & $0 \pm 0$ & $0.194-=0.112$ & $2.24 \pm 0.2$ \\
\hline $\begin{array}{l}\text { Maurolicus } \\
\text { mucronatus }\end{array}$ & Dragonfishes & Mesopelagic & KAEC & No & 10 & $0 \pm 0$ & $2.9-=2$ & $0 \pm 0$ & $0.188-=0.11$ & $2.34 \pm 0.25$ \\
\hline Vinciguerria mabahiss & Panama lightfish & Mesopelagic & KAEC & No & 10 & $0 \pm 0$ & $1.9=1.5$ & $0 \pm 0$ & $0.027--=0.014$ & $1.72 \pm 0.13$ \\
\hline
\end{tabular}




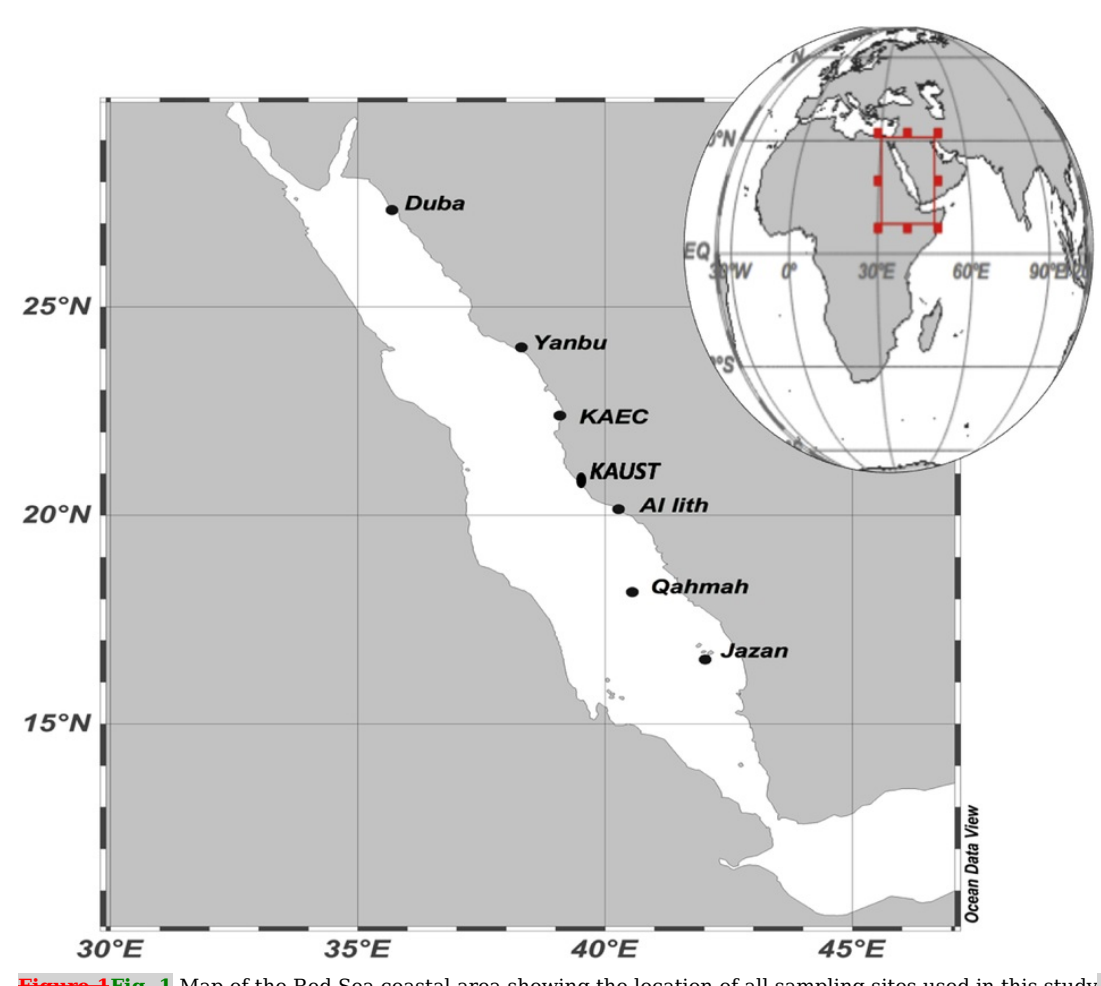

Figtre 1Fiq. 1 Map of the Red Sea coastal area showing the location of all sampling sites used in this study alt-text: Fig. 1

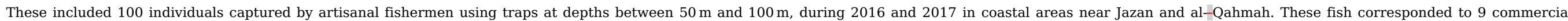

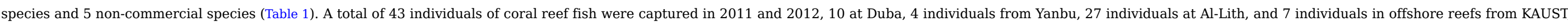

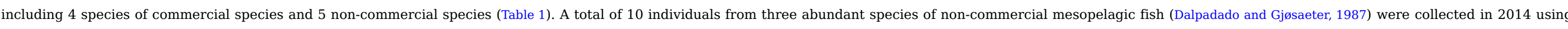

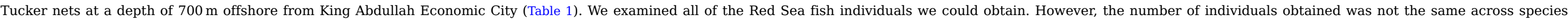
because of their different abundance, commercial interest and difficulty to catch (e.g. mesopelagic fish are notoriously difficult to catch).

\subsubsection{Sample preparation}

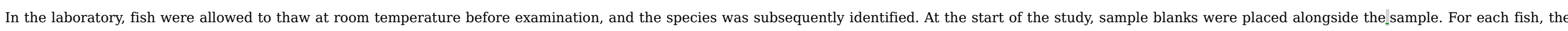
total length (TL) was taken (the length (cm), from the tip of the snout to the tip of the longer lobe of the caudal fin), and total wet weight ( $\mathrm{g}$ ) were measured prior to dissection.

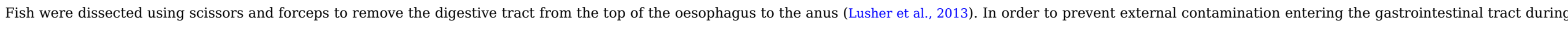

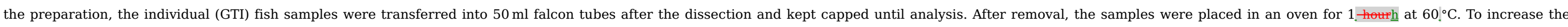

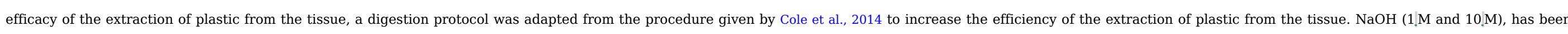

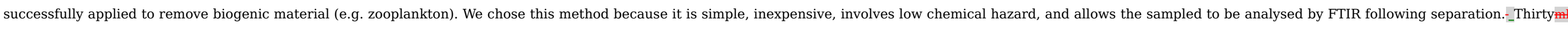

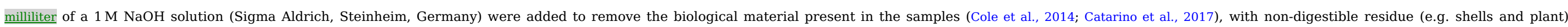
remaining, in addition to microplastic materials, following the chemical digestion. 


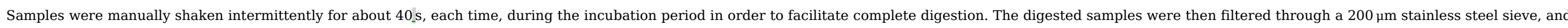

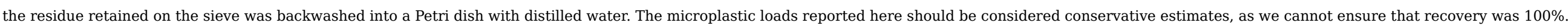

\subsubsection{Detection of microplastic}

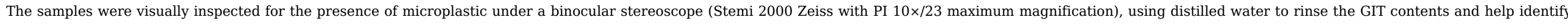

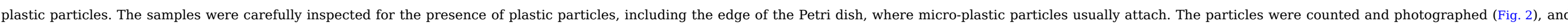
image-processing software "ImageJ" (v.1.50i;_http://imagej.nih.gov) was used to measure the maximum length of each particle (units mm)
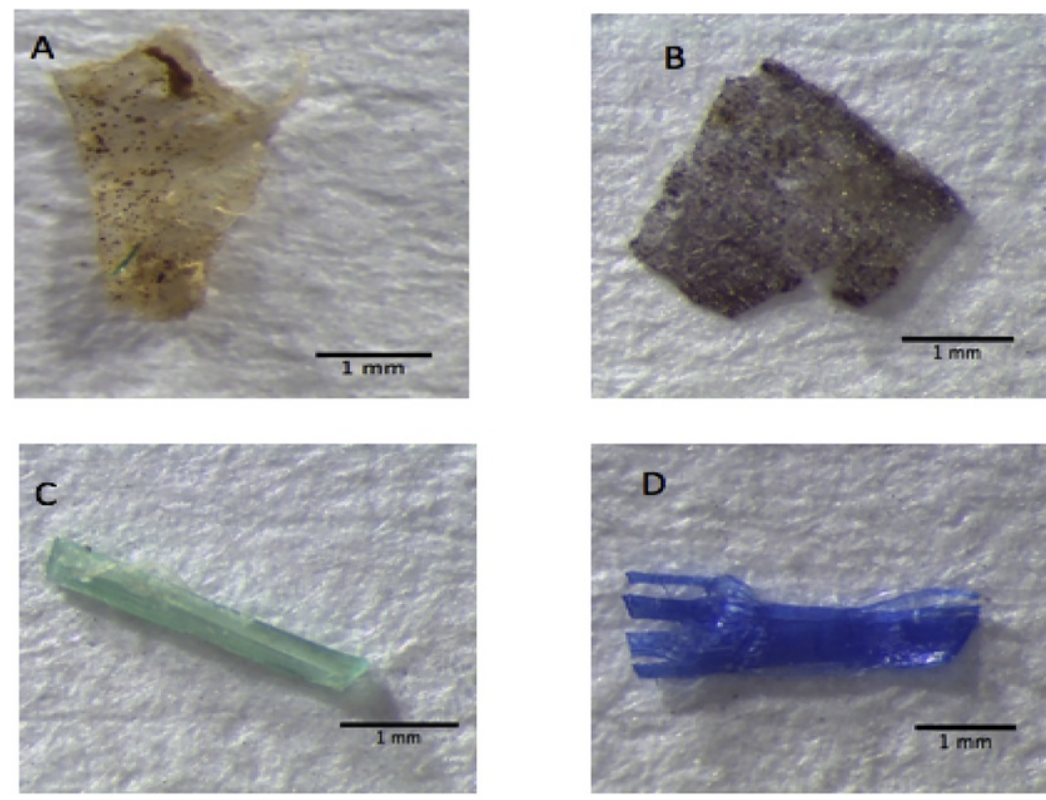

Figure 2Fiq. 2 The morphotypes included (A,/B) films (C,_D) fishing threads.

\section{alt-text: Fig. 2}

\subsubsection{FTIR polymer identification}

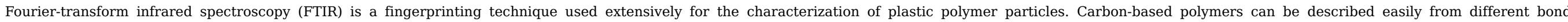

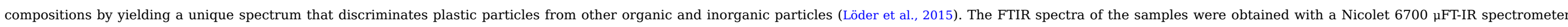

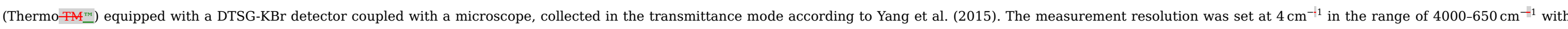

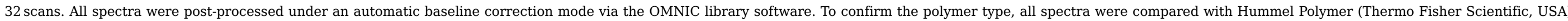

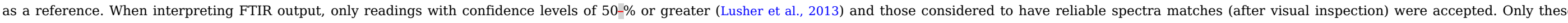

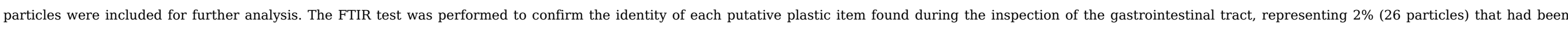

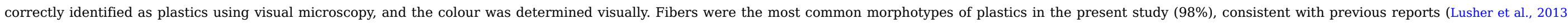

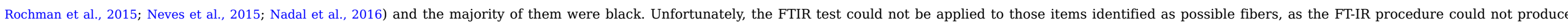

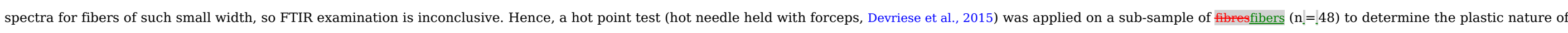

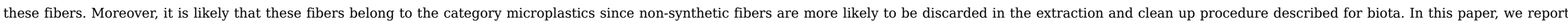




\subsubsection{Contamination prevention and blanks}

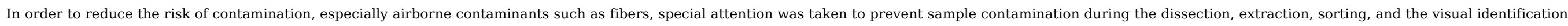

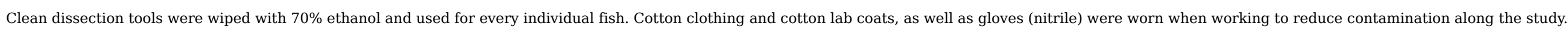

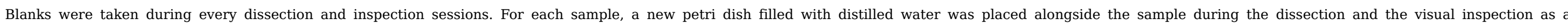

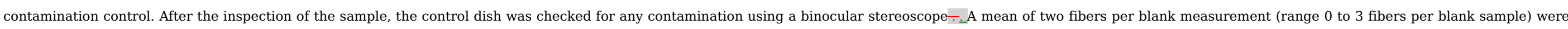

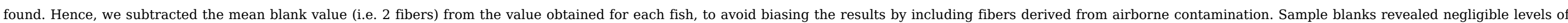
contamination, thus airborne contamination was not a risk in accordance with the corresponding fish sample.

\subsubsection{Statistical analyses}

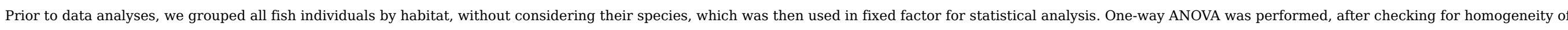

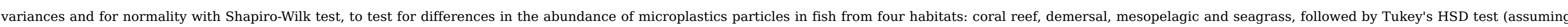

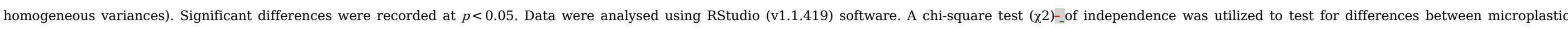
particle ingestion between fish species (commercial vs. non-commercial), with a significance level of $p<0.05$, conducted in SPSS v 1.0.0.800 (http://www-03.ibm.com/software).

\section{3 Results}

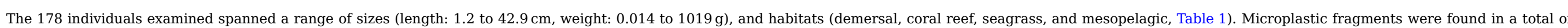

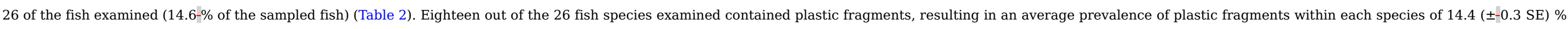

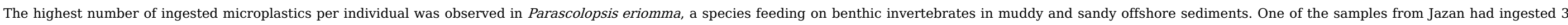

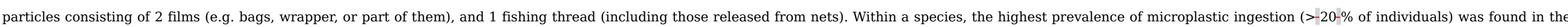

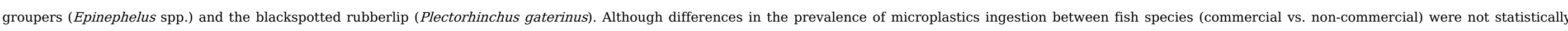
significant (Chi-square test; $\chi 23=6.04, p=0.109$ ).

Table 2 Frequency of microplastic ingestion per fish species

\begin{tabular}{|c|c|c|c|c|c|c|c|c|}
\hline Species name & Habitat & Location & Commercial__Yes/No & $\begin{array}{l}\text { Number of } \\
\text { stomachs } \\
\text { examined }\end{array}$ & $\begin{array}{l}\text { Number of microplastic found } \\
\text { in stomach per species }\end{array}$ & $\begin{array}{l}\text { Total number of-microplastic } \\
\text { found in stomach per species }\end{array}$ & $\begin{array}{l}\text { Average microplastic } \\
\text { size per species }(\mathrm{mm})\end{array}$ & $\begin{array}{c}\% \\
\text { Ingestion }\end{array}$ \\
\hline Acanthurus gahhm & Demersal & Jizan & Yes & 10 & 1 & 1 & 2.7 & 100 \\
\hline Pristipomoides typus & Demersal & Jizan & NO & 5 & 0 & 0 & 0 & 0 \\
\hline Epinephelus areolatus & Seagrass & Jizan & Yes & 5 & 1 & 1 & 1.8 & 20 \\
\hline Pristipomoides multidens & Demersal & Jizan/Qahmah & Yes & 10 & 2 & 2 & 3.8 & 20 \\
\hline Lutjanus kasmira & Coral reef & Jizan & Yes & 12 & 2 & 2 & 2.16 & 16.67 \\
\hline Lethrinus microdon & Coral reef & Jizan & Yes & 10 & 2 & 2 & 1.48 & 20 \\
\hline Gymnocranius grandoculis & Coral reef & Jizan & No & 10 & 2 & 2 & 2.35 & 20 \\
\hline Epinephelus chiorostigma & Seagrass & Jizan & Yes & 3 & 1 & 1 & 1.9 & 33.33 \\
\hline
\end{tabular}




\begin{tabular}{|c|c|c|c|c|c|c|c|c|}
\hline Parascolopsis eriomma & Demersal & Jizan & Yes & 5 & 3 & 3 & 1.38 & 60 \\
\hline Sargocentron spiniferum & Coral reef & Qahmah & NO & 5 & 0 & 0 & 0 & 0 \\
\hline Epinephelus radiatus & Demersal & Qahmah & NO & 7 & 1 & 1 & 2.14 & 14.29 \\
\hline Lipocheilus carnolabrum & Demersal & Qahmah & Yes & 7 & 2 & 2 & 1.87 & 28.57 \\
\hline Plectorhinchus gaterinus & Demersal & Qahmah & Yes & 6 & 2 & 2 & 3.31 & 33.33 \\
\hline Epinephelus epistictus & Demersal & Jizan & No & 5 & 1 & 1 & 2.71 & 20 \\
\hline Pygoplites diacanthus & Coral reef & $\begin{array}{l}\text { Offshore } \\
\text { KAUST }\end{array}$ & No & 5 & 0 & 0 & 0 & 0 \\
\hline Cephalopholis argus & Coral reef & Yanbu & Yes & 4 & 0 & 0 & 0 & 0 \\
\hline Abudefduf sexfasciatus & Coral reef & Al-Lith & No & 5 & 1 & 1 & 1.2 & 20 \\
\hline Acanthurus sohal & Coral reef & Al-Lith & Yes & 3 & 0 & 0 & 0 & 0 \\
\hline Dascyllus trimaculatus & Coral reef & Al-Lith & No & 2 & 0 & 0 & 0 & 0 \\
\hline Chaetodon austriacus & Coral reef & Duba & No & 10 & 1 & 1 & 4.68 & 100 \\
\hline Neoniphon sammara & Coral reef & Al-Lith & No & 5 & 1 & 1 & 1.51 & 20 \\
\hline Naso unicornis & Coral reef & $\begin{array}{l}\text { Offshore } \\
\text { KAUST }\end{array}$ & Yes & 2 & 0 & 0 & 0 & 0 \\
\hline Thalassoma rueppellii & Coral reef & Al-Lith & Yes & 12 & 1 & 1 & 1.93 & 8.33 \\
\hline Benthosema pterotum & Mesopelagic & KAEC & No & 10 & 1 & 1 & 2.58 & 100 \\
\hline Maurolicus mucronatus & Mesopelagic & KAEC & No & 10 & 1 & 1 & 1.42 & 100 \\
\hline Vinciguerria mabahiss & Mesopelagic & KAEC & No & 10 & 0 & 0 & 0 & 0 \\
\hline Total & & & & 178 & 26 & & & \\
\hline $\begin{array}{l}\text { Average of stomachs } \\
\text { with-microplastic per species }\end{array}$ & & & & & 1.00 & & & \\
\hline $\begin{array}{l}\text { Average by the total number of } \\
\text { fishes by species }\end{array}$ & & & & & 0.146 & & & \\
\hline
\end{tabular}

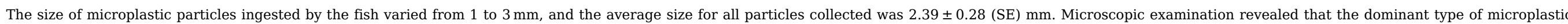

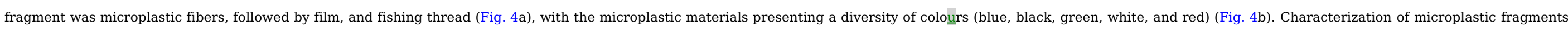
using FTIR spectroscopy showed that most of the particles were polypropylene, polyethylene, polystyrene, polyvinyl chloride, and polyacrylonitrile (Fig. 4c).

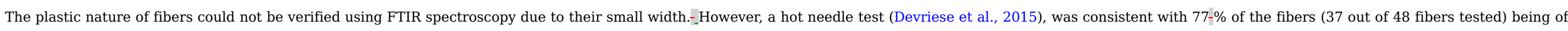
plastic materials.

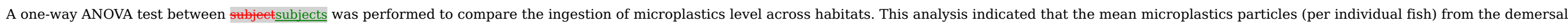
seagrass, and coral reef habitats were significantly higher than in the mesopelagic habitat (one-way ANOVA; $p<0.001 ; \mathrm{F}=13.83 ;$ Fig. 3) 


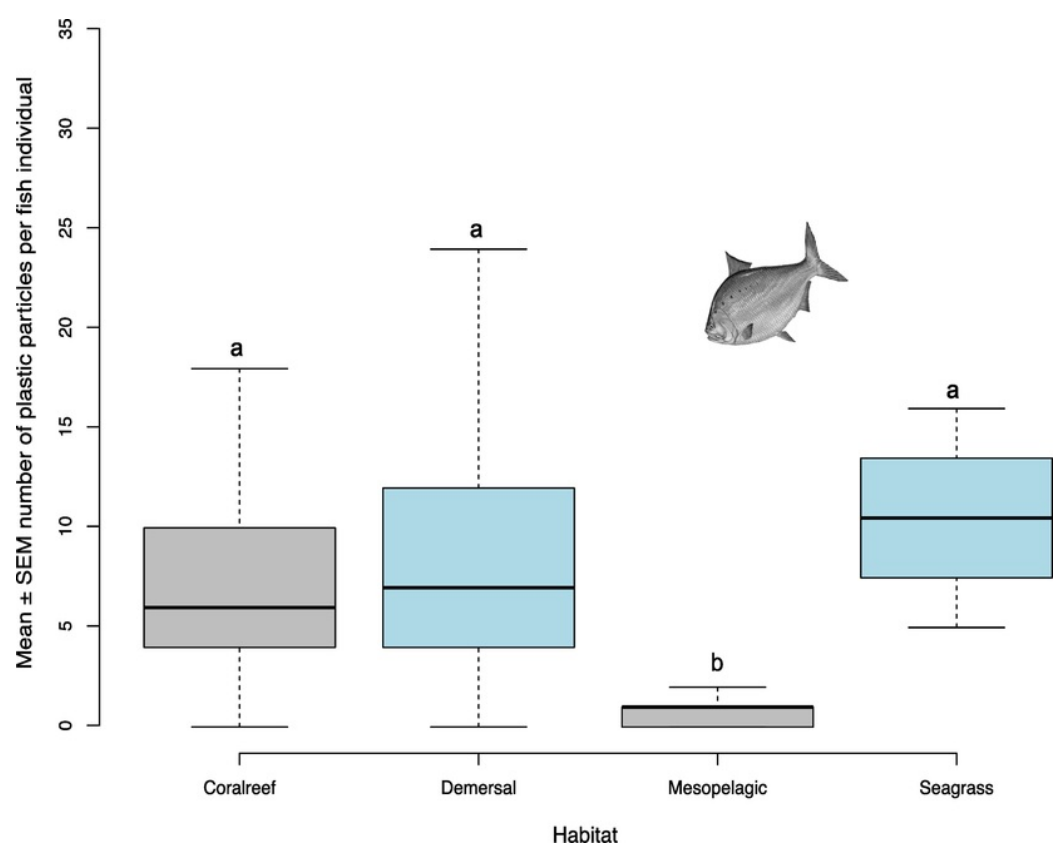

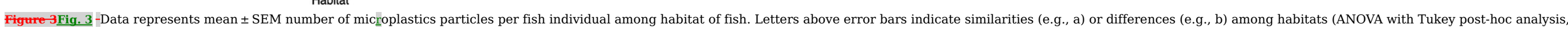
$p<0.05$ ).

alt-text: Fig. 3

(a)

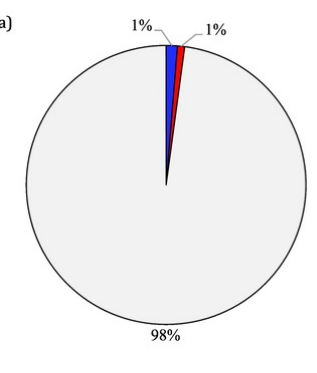

- Film

- Fishing thread

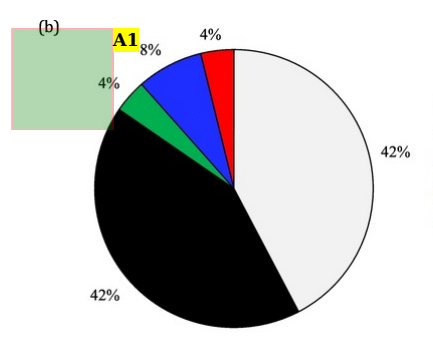

口Polypropylene
- Polyethylene

-Polyethylene
apolystyrene

- polyvinyl chloride

aFibers

- polyacrylonitrile

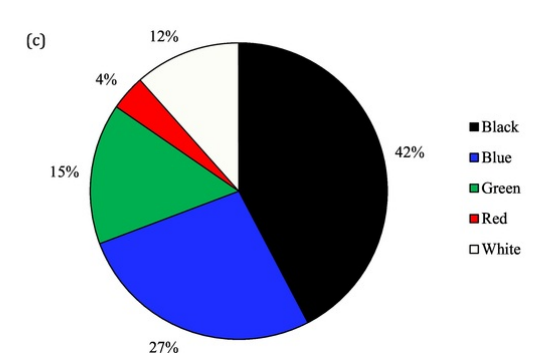

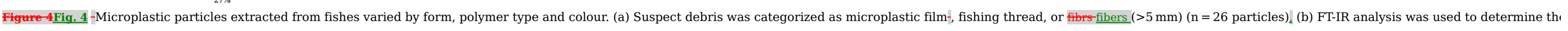

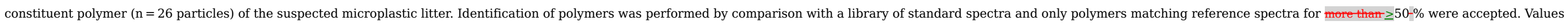
are expressed in percentages, (c) colour categories of plastic fragments in the stomach contents.(For interpretation of the references to color in this fiqure legend, the reader is referred to the web version of this article.) 


\section{Annotations:}

A1. the (b) and (a) need to be adjust as one show far (a) in the corner and (b)is up

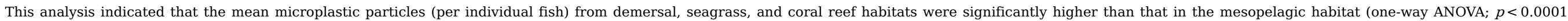
F = 13.83 Fig. 3). Indeed, mesopelagic fish were significantly smaller than fish sampled from other habitats.

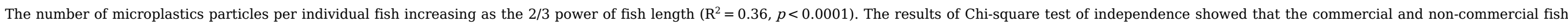
did not differ in the likelihood of containing microplastics items ( $\chi 23=6.04, p=0.109$ ), nor did the frequency of plastic abundance differ with trophic mode of the species (ANOVA, $p>0.05$ ).

\section{4. $\underline{4}$ Discussion}

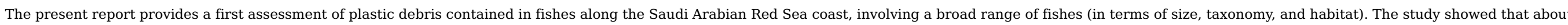

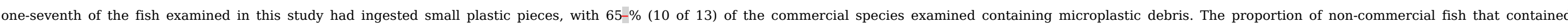

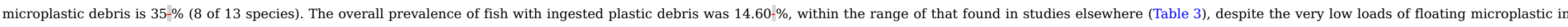

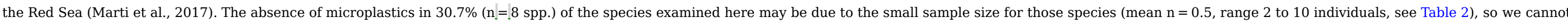
exclude the possibility that they would show a similar prevalence of marine microplastic in their guts if a larger sample size would have been obtained.

Table 3 Summary of the prevalence of plastic items found in fish in previous studies and the results reported here for Saudi Red Sea coast.

\section{alt-text: Table 3}

\begin{tabular}{|c|c|c|c|c|c|}
\hline Area & Type of fish & Sample & $\%$ Ingestion & Size of MP particles (mm) & Ref \\
\hline 1- North Pacific Subtropical Gyre & Mesopelagic & 141 & $9.20 \%$ & $2.2 \pm 1.9$ & Davison and Asch (2011) \\
\hline 2- Portuguese coast & Commercial & 263 & $32.70 \%$ & $2.11 \pm 1.67$ & Neves et al. (2015) \\
\hline 3- English Channel & Pelagic and demersal & 504 & $36.50 \%$ & $0.13 \pm 14.3$ & Lusher et al. (2013) \\
\hline 4- Mediterranean Sea & Pelagic fish & 121 & $18.2 \%$ & $1.51 \pm \pm 16.50$ & Romeo et al. (2015) \\
\hline 5-Swedish west coast & Demersal fish & 62 & $68 \%$ & $=-$ & Karlsson et al. (2017) \\
\hline 6- Northwest Atlantic & Mesolelagie-Mesopelagic fish & 280 & $73 \%$ & $969 \pm 1048$ & Wieczorek et al. (2018) \\
\hline 7- French -rivers & Wild fish & 186 & $12 \%$ & $=$ & Sanchez et al. (2014) \\
\hline 8- Adriatic Sea & Commercial & 125 & $28 \%$ & $1.78 \pm 0.97$ & Avio et al. (2015) \\
\hline 9-_Red Sea & Commercial and non--commercial & 178 & $14.60 \%$ & $2.39 \pm 0.28$ & This study \\
\hline
\end{tabular}

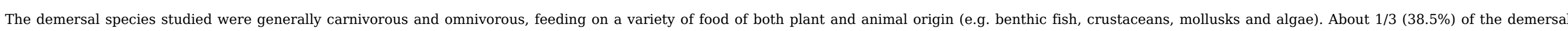
species examined had at least one individual with ingested microplastics.

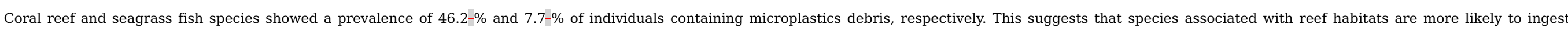

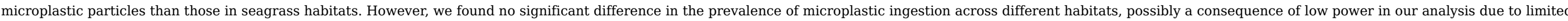

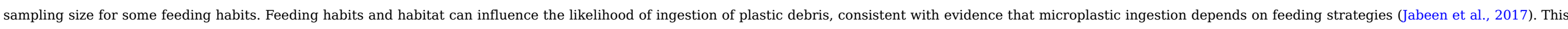
suggests that ingestion of microplastic might occur depending on the feeding habits of fish regardless of prey type.

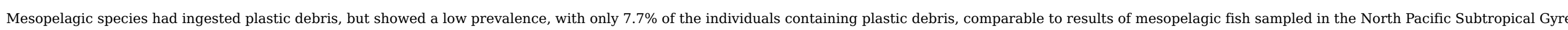




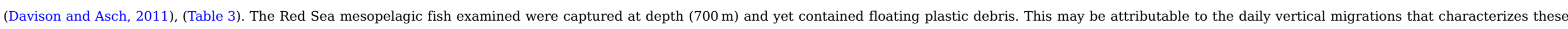

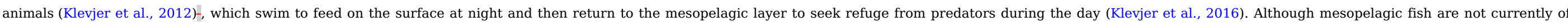

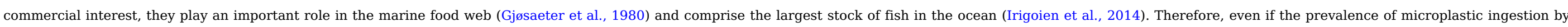

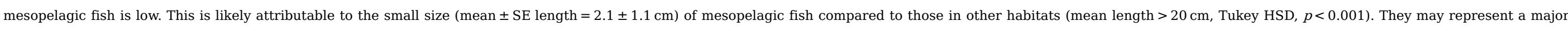

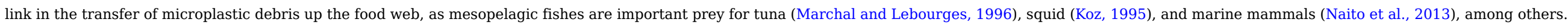

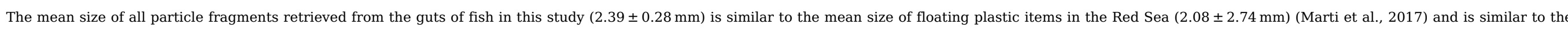

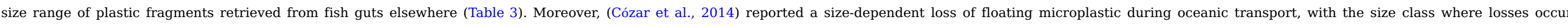

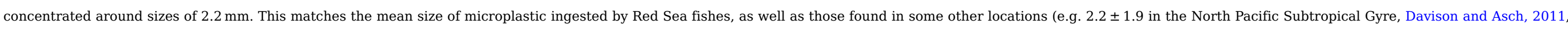

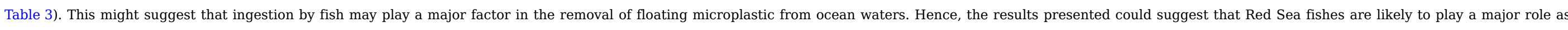
sinks of floating microplastics, which would lead to the transference of microplastic along the food web, to which human are connected.

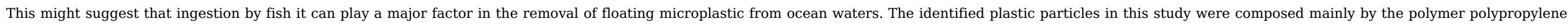

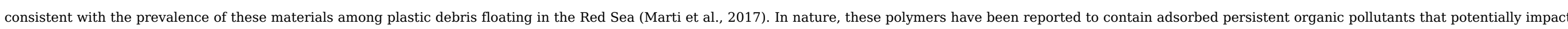

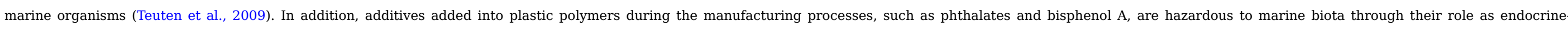

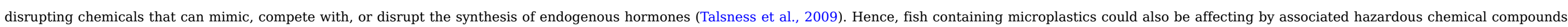
with possible impacts on fish health transferred along the food web.

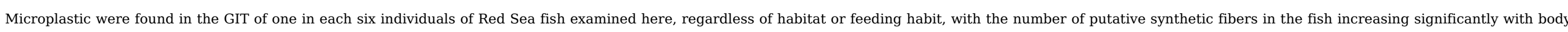

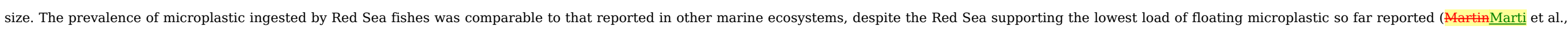

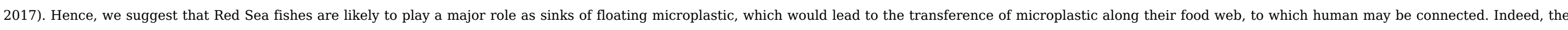

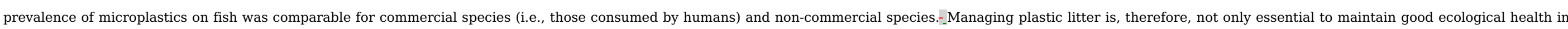
the Red Sea and elsewhere, but also to protect consumers from ingesting the plastic we dispose in the environment (Koelmans et al., 2017).

\section{5. $\underline{5}$ Conclusion}

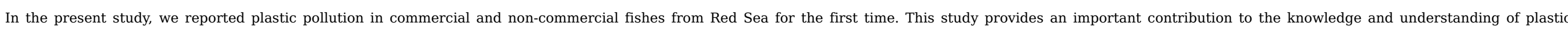
occurrence in these commercial and non-commercial fish, given also their importance in Red Sea catches and human consumption.

\section{Acknowledgements}

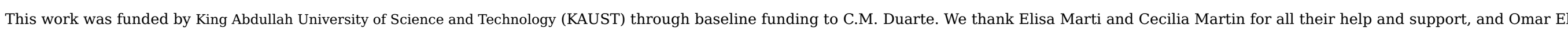
Tall for his assistance with FTIR and technical support.

\section{References}

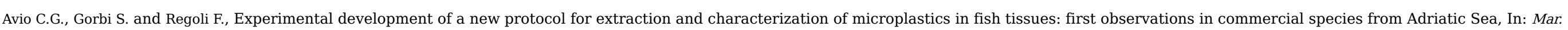
Environ. Res., Particles in the Oceans: Implication for a Safe Marine Environment vol. 111, 2015, 18-26.

Cannon S.M.E., Lavers J.L. and Figueiredo B., Plastic ingestion by fish in the Southern Hemisphere: a baseline study and review of methods, Mar. Pollut. Bull. 107, $2016,286-291$.

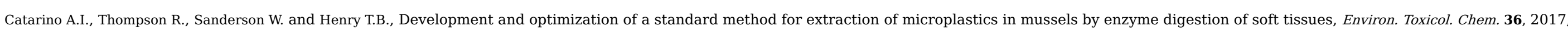
947-951.

Cole M., Webb H., Lindeque P.K., Fileman E.S., Halsband C. and Galloway T.S., Isolation of microplastics in biota-rich seawater samples and marine organisms, Sci. Rep. 4, 2014. 


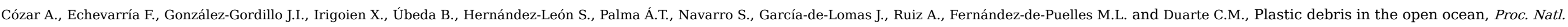
Acad. Sci. 111, 2014, 10239-10244.

Cózar A., Sanz-Martín M., Martí E., González-Gordillo J.I., Ubeda B., Gálvez J.Á., Irigoien X. and Duarte C.M., Plastic accumulation in the Mediterranean Sea, PLOS ONEPLoS One 10, 2015 , e0121762.

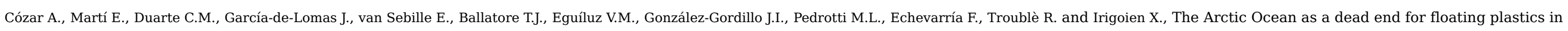
the North Atlantic branch of the Thermohaline Circulation, Sci. Adv. 3, 2017, e1600582.

Davison P. and Asch R.G., Plastic ingestion by mesopelagic fishes in the North Pacific Subtropical Gyre, Mar. Ecol. Prog. Ser. 432, 2011, 173-180.

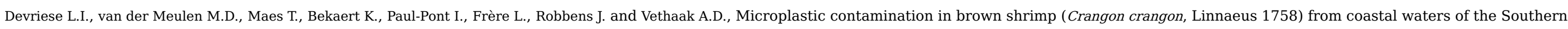
North Sea and Channel area, Mar. Pollut. Bull. 98, 2015, 179-187.

Dalpadado P. and Gjøsaeter J., Observations on mesopelagic fish from the Red Sea, Mar. Biol. 96, 1987, 173-183.

Gjøsaeter J., Kawaguchi K., Nations F. and A.O. of the U, A Review of the World Resources of Mesopelagic Fish, 1980, Food \& Agriculture Org..

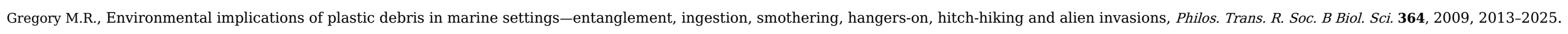

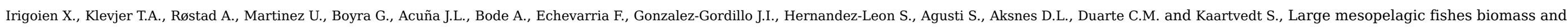
trophic efficiency in the open ocean, Nat. Commun. 5, 2014, 3271.

Jabeen K., Su L., Li J., Yang D., Tong C., Mu J. and Shi H., Microplastics and mesoplastics in fish from coastal and fresh waters of China, Environ. Pollut. 221, 2017, $141-149$.

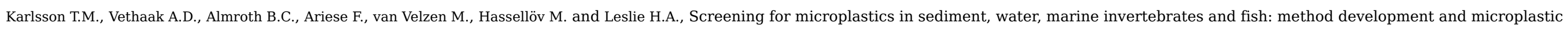
accumulation, Mar. Pollut. Bull. 122, 2017, 403-408.

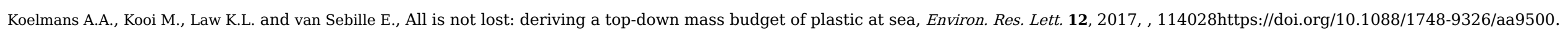

Koz A., A review of the trophic role of mesopelagic fish of the family Myctophidae in the Southern Ocean ecosystem, CCAMLR SCi. 2, $1995,71-77$.

Klevjer T.A., Torres D.J. and Kaartvedt S., Distribution and diel vertical movements of mesopelagic scattering layers in the Red Sea, Mar. Biol. 159, 2012, 1833-1841.

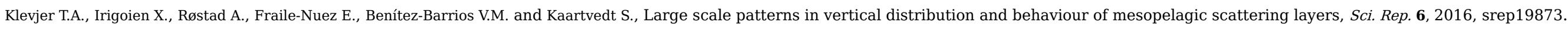

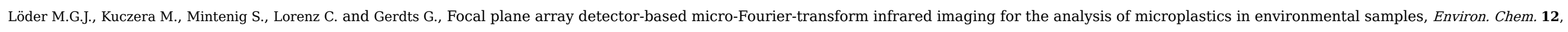
$2015,563-581$.

Lusher A.L., McHugh M. and Thompson R.C., Occurrence of microplastics in the gastrointestinal tract of pelagic and demersal fish from the English Channel, Mar. Pollut. Bull. 67, 2013, 94-99.

Marchal E. and Lebourges A., Acoustic evidence for unusual diel behaviour of a mesopelagic fish (Vinciguerria nimbaria) exploited by tuna, ICES J. Mar. Sci. 53, $1996,443-447$.

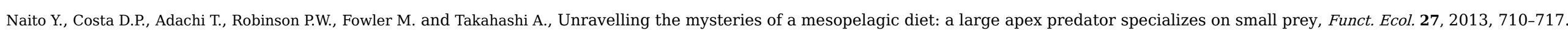

Nadal M.A., Alomar C. and Deudero S., High levels of microplastic ingestion by the semipelagic fish bogue Boops boops (L.) around the Balearic Islands, Environ. Pollut. 214, $2016,517-523$.

Neves D., Sobral P., Ferreira J.L. and Pereira T., Ingestion of microplastics by commercial fish off the Portuguese coast, Mar. Pollut. Bull. 101, 2015, 119-126.

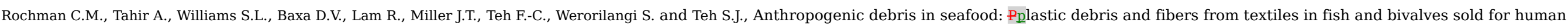
consumption, Sci. Rep. 5, 2015.

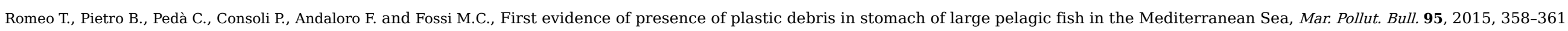




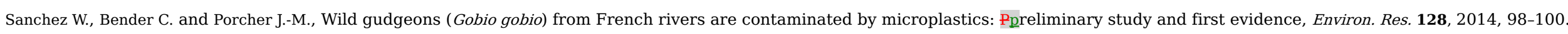

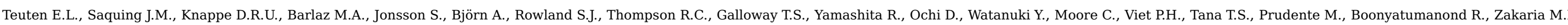

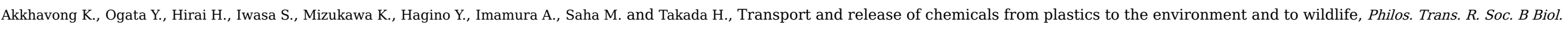

Sci. 364, 2009, 2027-2045.

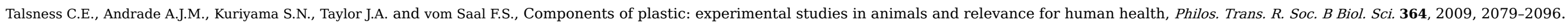

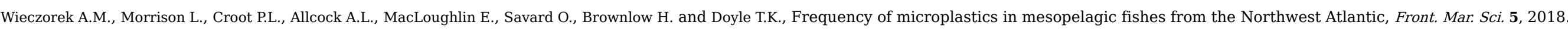

\section{Highlights}

- Microplastics pose a risk to organisms,

- Plastic litter in fishes from different habitat types (coral reef, demersal, mesopelagic and seagrass) were assessed.

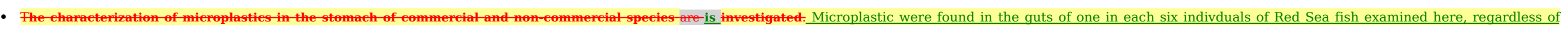
habitat or feeding.

- The majority of plastics were fibers, a limited number of particles has scored.

\section{Queries and Answers}

Query:

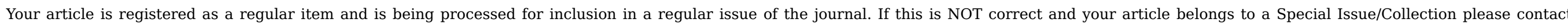
l.barker@elsevier.com immediately prior to returning your corrections.

Answer: Yes

Query:

Please confirm that given names and surnames have been identified correctly and are presented in the desired order, and please carefully verify the spelling of all authors' names.

Answer: Yes

Query:

The author names have been tagged as given names and surnames (surnames are highlighted in teal color). Please confirm if they have been identified correctly.

Answer: Yes

Query:

Citation "Andrady and Neal, 2009" has not been found in the reference list. Please supply full details for this reference.

Answer: Andrady, A.L., Neal, M.A., 2009. Applications and societal benefits of plastics. Philos. Trans. R. Soc. B Biol. Sci. 364, $1977-1984$.

Query: 
Citation "Sigler, 2014" has not been found in the reference list. Please supply full details for this reference.

Answer: Sigler, M., 2014. The Effects of Plastic Pollution on Aquatic Wildlife: Current Situations and Future Solutions. Water Air Soil Pollut $225,2184$.

Query:

Citation "Ryan et al., 2009" has not been found in the reference list. Please supply full details for this reference.

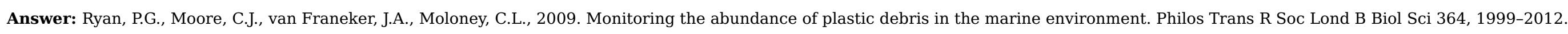

Query:

Citation "Li et al., 2016" has not been found in the reference list. Please supply full details for this reference.

Answer: Li, W.C., TSE, H.F., FOK, L., 2016. Plastic waste in the marine environment: A review of sources, occurrence and effects. Sci. Total Environ. 566, 333-349.

Query:

Citation "Zitko and Hanlon, 1991" has not been found in the reference list. Please supply full details for this reference.

Answer: Zitko, V., Hanlon, M., 1991. Another source of pollution by plastics: Skin cleaners with plastic scrubbers. Mar. Pollut. Bull. 22 , 41-42.

Query:

Citation “Lusher et al., 2015" has not been found in the reference list. Please supply full details for this reference.

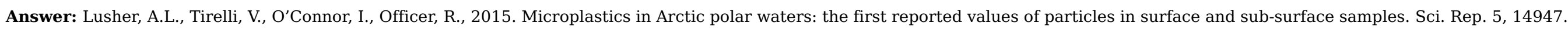

Query:

Citation "Sul et al., 2014" has not been found in the reference list. Please supply full details for this reference.

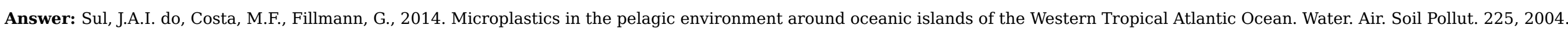

Query:

Citation "Marti et al., 2017" has not been found in the reference list. Please supply full details for this reference.

Answer: Marti, E., Martin, C., Cózar, A., Duarte, C., 2017. Low Abundance of Plastic Fragments in the Surface Waters of the Red Sea. Frontiers in Marine Science 4, 333.

Query:

Citation "Yang et al. (2015)" has not been found in the reference list. Please supply full details for this reference.

Answer: Yang, D., Shi, H., Li, L., Li, J., Jabeen, K., Kolandhasamy, P., 2015. Microplastic pollution in table salts from China. Environ. Sci. Technol. 49 (22):13622-13627.

Query:

Citation "Martin et al., 2017" has not been found in the reference list. Please supply full details for this reference.

Answer: Marti, E., Martin, C., Cózar, A., Duarte, C., 2017. Low Abundance of Plastic Fragments in the Surface Waters of the Red Sea. Frontiers in Marine Science 4, 333.

Query:

Please provide the corresponding grant number(s) for the following grant sponsor(s): "King Abdullah University of Science and Technology". 
Answer: there is no grant number. 